\title{
Uveitis Glaucoma And Hyphema Syndrome
}

National Cancer Institute

\section{Source}

National Cancer Institute. Uveitis Glaucoma And Hyphema Syndrome. NCI Thesaurus.

Code C50794.

Clinical triad of uveitis, glaucoma, and hyphema (UGH), especially associated with the early anterior chamber intraocular lens (IOL).Glaucoma is believed to be caused by movement of the IOL against the iris causing release of inflammatory and red blood cell debris, which obstruct the trabecular meshwork. The haptic also may cause direct damage to the trabecular meshwork contributing to the glaucoma. Incidence of UGH is dependent on both surgical methodology applied in the cataract population and type of IOL used. UGH is particularly common if metal clip lenses have been used. Components of the condition may be reversed if the offending IOL is removed before permanent damage has occurred. 